\title{
The Effect of LINC01296 Expression in Patients with Cancer: A Systematic Review and Meta-Analysis
}

\author{
Farzane Saeidi ${ }^{1}$, Kiarash Tanha ${ }^{2}$, Mostafa Davoodabadi Farahani ${ }^{3}$, Ehsan \\ Sohrabi $^{4}$, Yousef Moradi ${ }^{5,6 *}$, Pouria Khani ${ }^{7 *}$
}

\begin{abstract}
Background: Recently has been suggested that LINC01296 has an important role in tumor-promoting in different malignancies. We performed first meta-analysis to assess the association between the LINC01296 expression and clinicopathological criteria and the survival of patients with cancers. Methods: Relevant articles Identified by PubMed, EMBASE, Web of Science, and Scopus searching between December 2000 and 28 December 2018. Binomial data were evaluated by the odds ratio (OR) as the rapid statistic. The association between overall survival (OS) and the LINC01296 expression was evaluated using pooling the hazard ratio (HR) with its corresponding 95\% confidence interval (CI). Results: Finally, 9 studies with 720 patients with cancer were included. The expression of LINC01296 showed a significant positive association with $\mathrm{TNM}$ stage $(\mathrm{OR}=2.67,95 \% \mathrm{CI}=1.83-3.88)$, tumor stage $(\mathrm{OR}=2.22,95 \% \mathrm{CI}=$ 1.34-3.66) and lymph node metastasis $(\mathrm{OR}=3.07,95 \% \mathrm{CI}=2.23-4.21)$. A shorter OS was significantly associated with the expression of $\operatorname{LINC} 01296(\mathrm{HR}=3.95,95 \% \mathrm{CI}=2.65-5.25)$ and lymph node metastasis $(\mathrm{HR}=2.39,95 \% \mathrm{CI}$ $=1.16-3.63)$. The OS did not show significant association with gender $(\mathrm{HR}=0.83,95 \% \mathrm{CI}=-0.63-2.30)$ and tumor stage $(\mathrm{HR}=2.66,95 \% \mathrm{CI}=-0.22-5.54)$. Conclusion: In conclusion, the results of this meta-analysis suggest that the expression of LINC01296 might be considered as a potential biomarker in patients with cancer.
\end{abstract}

Keywords: LINC01296- survival- cancer- biomarker

Asian Pac J Cancer Prev, 21 (8), 2189-2195

\section{Introduction}

More than $90 \%$ of the genome of human is transcribed as non-coding RNAs (ncRNAs). This subject suggests that ncRNAs as functional RNA molecules might involve in regulation of gene expression at the transcriptional and post-transcriptional levels in human cells (Ponting and Belgard, 2010; Siegel et al., 2014; Kurozumi et al., 2017). Short ncRNAs (eg microRNAs or miRNAs) and long ncRNAs (or IncRNA) are two broad categories of ncRNAs and in recent years have been revealed to have key functions in various biological processes (i.e., growth, differentiation) especially in cancers (Kurozumi et al., 2017; Monteleone and Lutz, 2017). IncRNAs are a various class of ncRNAs with more than 200 nucleotides in length (Bartonicek et al., 2016). They can be located within introns, between genes or be transcribed as natural antisense of coding genes and play prominent roles in development of various normal cells and diseases progression (Derrien et al., 2012; Kung et al., 2013). Aberrant expression and dysregulation of lncRNAs such as PCGEM1, SPRY4-IT1, PANDAR, and ROR has been shown in malignant cells (Srikantan et al., 2000; Jiang et al., 2017; Liu et al., 2017; Xu et al., 2018). According to the position and transcription direction, lncRNAs are categorized into subgroups including antisense, overlapping, intergenic, intronic, bidirectional, and processed (Bartonicek et al., 2016). Given that understanding of involved non-coding RNAs in cancer pathogenesis could contribute to better treatment of cancer (Xu et al., 2017).

Long intergenic non-protein-coding RNA 1296 (LINC01296) is mapped to $14 \mathrm{q} 11.2$ and plays an important role in several types of cancers. LINC01296 has been suggested acts as a tumor-promoting in different malignancies including gastric cancer(Qin et al., 2018), prostate cancer (Wu et al., 2017), colorectal cancer (Qiu and Yan, 2015). Up regulation of LINC01296 in

${ }^{1}$ Department of Medical Genetics, School of Medical Sciences, Tarbiat Modares University, Tehran, Iran. ${ }^{2}$ Department of Biostatistics, School of Public Health, Iran University of Medical Sciences, Tehran, Iran. ${ }^{3}$ Department of Medical Genetics, Faculty of Medicine, Shiraz University of Medical Sciences (SUMS), Shiraz, Iran. ${ }^{4}$ Department of Medical Genetics and Molecular Biology, Faculty of Medicine, Iran University of Medical Sciences (IUMS), Tehran, Iran. ${ }^{5}$ Department of Epidemiology, School of Public Health, Iran University of Medical Sciences, Tehran, Iran. ${ }^{6}$ Social Determinants of Health Research Center, Research Institute for Health Development, Kurdistan University of Medical Sciences, Sanandaj, Iran. ${ }^{7}$ Department of Medical Genetics, Faculty of Medicine, Tehran University of Medical Sciences (TUMS), Tehran, Iran.*For Correspondence: Pouriakhani70@gmail.com 
gastric cancer cells and cell lines resulted to aggravate GC development using the miR-122/MMP-9 axis (Qin et al., 2018). It has been shown LINC01296 silencing could suppress proliferation of prostate cancer cell and its invasion. Moreover, its expression could be introduced as a biomarker for survival analysis in prostate cancer patients (Wu et al., 2017). Overexpression LINC01296 was associated with poor prognosis of patients with colorectal cancer (Qiu and Yan, 2015). Up regulation of LINC01296 in pancreatic ductal adenocarcinoma tissues and non-small cell lung cancer was related to cell growth and progression, clinical characteristics and poor prognosis (e.g. lymph node metastasis and increasing in TNM stage)(Xu et al., 2019; Yuan et al., 2019).

In this study, we hypothesized that LINC01296 might influence the survival of patients with various cancers. There is no meta-analysis has been implemented to assess the correlation between LINC01296 and the survival of patients with cancers. Hence, we decided to perform this meta-analysis study.

\section{Materials and Methods}

\section{Study Design}

This article was reported according to recommended guidelines Cochrane library for systematic reviews; the Preferred Reporting Items for Systematic Reviews and Meta-analysis (PRISMA) (Moher et al., 2009a).

\section{Search strategy}

Identification of relevant articles for this study performed by searches of PubMed, EMBASE, Web of Science, and Scopus between December 2000 and 28 December 2018. The electronic search terms were [LINC01296 and Cancer (with special MeSH terms for PubMed and Emtree terms for Embase)]. Manual search was performed in reference list and citations of included studies as well as related websites. Two authors (PKH and FS) were cooperated in all stages. Differences were discussed until agreement was reached. It was necessary to contact study author for several times. Country was not limited in this search strategy.

\section{Inclusion and exclusion criteria}

We included studies that meet the subsequent criteria: (1) case control and cohort studies that evaluate the correlation between the clinicopathological parameters and LINC01296 in patients with cancer; (2) cancer should be diagnosed according to histopathological evaluation; (3) None of the patients had undergone radiotherapy or chemotherapy treatment before surgery (4) full articles published in English. In this study we excluded: (1) the studies that solely were related to cancer or LINC01296; (2) animal study; (3) the study was a review or abstract; (4) Studies whose information was inaccessible.

\section{Data Extraction and Risk of Bias}

Two authors (PKH and FS) independently reviewed by title, abstract and full text and extracted the data from identified eligible studies. Probable discrepancies between two independent experts were resolved under the supervision of the main investigator (YM). The following data were extracted: the first author's name first author; year of publication; the patient gender; site of cancer and tumor type; tumor stage; sample size and covariants; outcome; type of treatment interval; experimental techniques used. Finally, we using the Newcastle-Ottawa Scale (NOS) assessed the methodological quality of included studies (Wells, 2001).

\section{Statistical analysis}

Binomial data were evaluated by the odds ratio (OR) as the rapid statistic. The correlation between LINC01296 and survival of cancer was evaluated by combining the HR with its corresponding 95\% CI. The heterogeneity of studies examined by $\mathrm{I} 2$ statistic and the chi-squared based Q-statistic test. The fixed effect models were used to estimate the pooled effect sizes across studies. Determination of all the P-values was performed using a 2-sided test. Software for all statistical analyses was STATA software version 13.

\section{Ethics statement \\ Consent to participate was unnecessary.}

\section{Results}

\section{Study characteristics}

Figure 1 show the PRISMA flow diagram (Moher et al., 2009b) of study identification of relevant studies. Fifty-seven studies were found in the preliminary search. After a comprehensive evaluation comprises remove of duplicates, exclusion based on title or abstract and exclusion based on some reasons such as inaccessibility to their data, finally, 9 studies with 720 patients with cancer were included in this meta-analysis. Pancreatic ductal adenocarcinoma (PDAC), non-small cell lung cancer, Esophageal squamous cell carcinoma (ESCC), Cholangiocarcinoma, breast cancer, urothelial carcinoma of the bladder, prostate cancer, gastric cancer was studied in the original studies. The characteristics of the included studies have been shown in Table 1. The data of Overall Survival (OS) extracted from Only 5 studies.

\section{Results of the meta-analysis}

Table 2 shows the associations between the clinicopathological criteria and the LINC01296 expression. No significant association was identified between the LINC01296 expression and gender (OR $=1.10$, 95\% $\mathrm{CI}=0.78-1.53$ ) (Figure 2 and Table 2). Moreover, the LINC01296 expression was not related to the patients age $(\mathrm{OR}=1.31,95 \% \mathrm{CI}=0.96-1.8)$ (Table 2). Conversely, the expression of LINC01296 showed a significant positive association with TNM stage $(\mathrm{OR}=2.67,95 \%$ $\mathrm{CI}=1.83-3.88)($ Table 2$)$, tumor stage $(\mathrm{OR}=2.22,95 \%$ $\mathrm{CI}=1.34-3.66)$ (Table 2 and Figure 3 ) and lymph node metastasis (invasion) $(\mathrm{OR}=3.07,95 \% \mathrm{CI}=2.23-4.21)$ (Figure 4). Additionally, a shorter OS (overall survival) in patients was significantly associated with the expression of this RNA (HR $=3.95,95 \% \mathrm{CI}=2.65-5.25)$ and lymph node metastasis $(\mathrm{HR}=2.39,95 \% \mathrm{CI}=1.16-3.63)$ (Table 2$)$. The OS did not show significant association with gender 


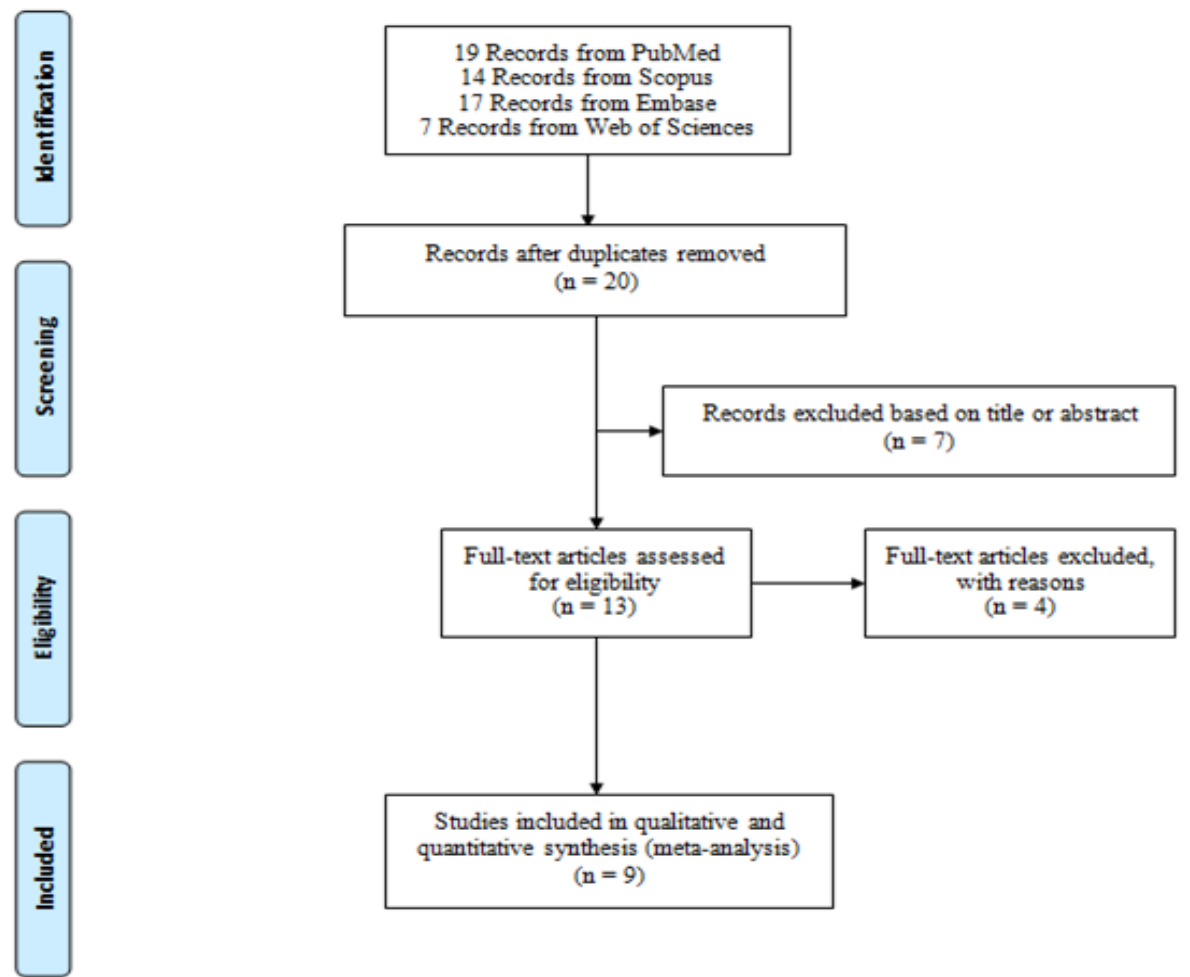

Figure 1. PRISMA Flow Diagram of Study Identification

$(\mathrm{HR}=0.83,95 \% \mathrm{CI}=-0.63-2.30)$ and tumor stage $(\mathrm{HR}=$ $2.66,95 \% \mathrm{CI}=-0.22-5.54)$ (Table 2).

Meta Regression

Meta regression was used to explore the sources of heterogeneity among studies, including age and gender. The results of this this analysis showed that the associations are not related to age $(\mathrm{B}=-0.00043, \mathrm{P}=0.345$, $95 \% \mathrm{CI}-0.007,0.0010)$ and gender $(\mathrm{B}=0.0380, \mathrm{P}=0.478$, $95 \%$ CI $-0.046,0.022)$.

\section{Publication Bias Assessment}

The results of Egger's test show no significant bias occurred in the publication of the results (Egger's test $=$ 1.01 SE: $0.221, \mathrm{P}=0.443$ ).

\section{Discussion}

Recently, several studies have demonstrated abnormal expression of LINC01296 has been associated with several malignancies (Jiang et al., 2018). Therefore, based on information provided by several studies, we performed the first meta-analysis to assess the correlation between the LINC01296 expression and prognosis based on patients clinicopathological parameters in cancers. The results of our meta-analysis demonstrated that the expression of LINC01296 was significantly related with lymph node metastasis, TNM stage and Tumor stage. Moreover,

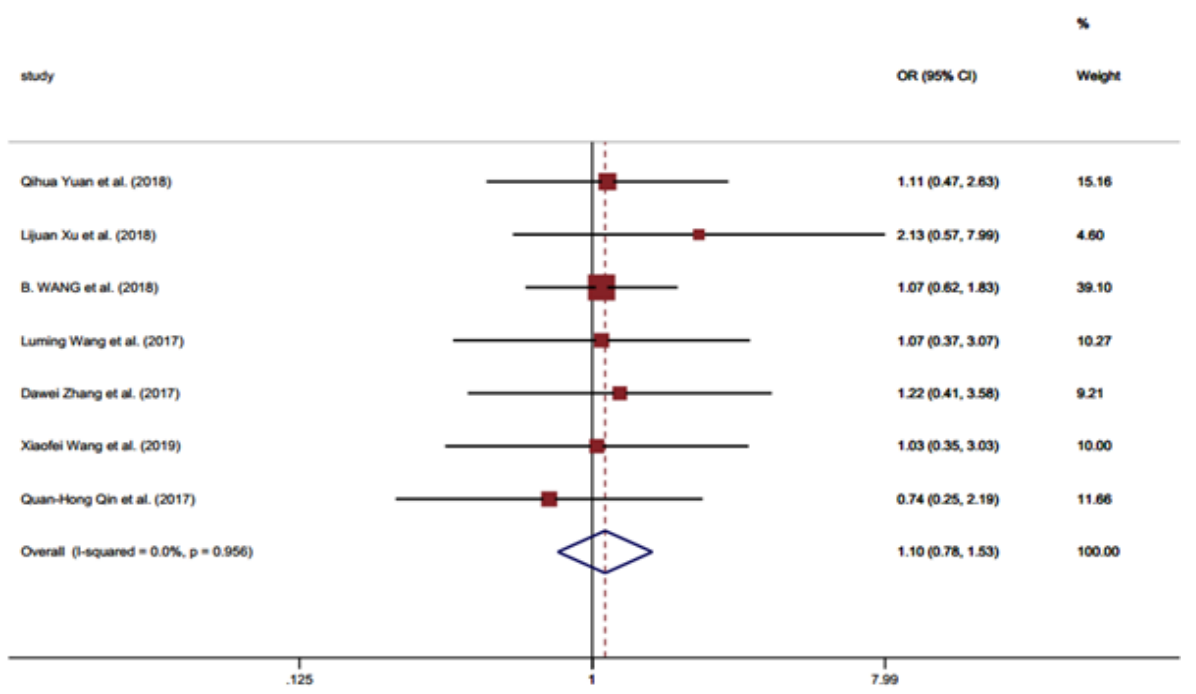

Figure 2. Forest Plot Illustrating the Association between the LINC01296 Expression and Gender of Patients 
Table 1. Features of the Included Studies

\begin{tabular}{|c|c|c|c|c|c|c|c|c|c|}
\hline First Author & $\begin{array}{l}\text { Year of } \\
\text { publica- } \\
\text { tion }\end{array}$ & $\begin{array}{l}\text { Gender } \\
\text { ( Male / } \\
\text { Female) }\end{array}$ & Type of cancer & $\begin{array}{l}\text { TNM } \\
\text { stage }\end{array}$ & $\begin{array}{l}\text { Sample } \\
\text { Size }\end{array}$ & Outcome & $\begin{array}{l}\text { Lymph node } \\
\text { metastasis } \\
\text { (Present / } \\
\text { Absent) }\end{array}$ & $\begin{array}{l}\text { Tumor } \\
\text { Stage }\end{array}$ & NOS \\
\hline Yuan, Q., et al. & 2018 & $47 / 38$ & Pancreatic ductal adenocarcinoma & NA & 85 & OS & $53 / 32$ & $\mathrm{~T} 1-4$ & 7 \\
\hline Xu, L., et al. & 2018 & $26 / 14$ & NSCLC & I-IV & 40 & OS* & $20 / 20$ & NA & 7 \\
\hline Wang, B., et al. & 2018 & $134 / 87$ & Esophageal squamous cell carcinoma & I-IV & 221 & OS & $83 / 138$ & NA & 7 \\
\hline Wang, L., et al. & 2017 & $60 / 18$ & Esophageal squamous cell carcinoma & I-IV & 78 & OS* & $27 / 51$ & $\mathrm{~T} 1-4$ & 7 \\
\hline Zhang, D., et al. & 2017 & $32 / 25$ & Cholangiocarcinoma & I-IV & 57 & OS* & $28 / 29$ & NA & 7 \\
\hline Jiang, M., et al. & 2018 & 55 (Female) & Breast cancer & I-III & 55 & OS & $36 / 19$ & NA & 7 \\
\hline Wang, X., et al. & 2019 & $30 / 24$ & Urothelial carcinoma of the bladder & NA & 54 & OS & $18 / 36$ & NA & 7 \\
\hline Wu, J., et al. & 2017 & 73 (Male) & Prostate cancer & NA & 70 & OS & $38 / 32$ & $\mathrm{~T} 2-4$ & 7 \\
\hline Qin, Q.H., et al. & 2017 & $33 / 27$ & Gastric cancer & I-III & 60 & OS* & $48 / 12$ & $\mathrm{~T} 1-3$ & 7 \\
\hline
\end{tabular}

OS, overall survival; OS*, no data of OS could be extracted; NOS, Newcastle-Ottawa Scale; NA, not available; NSCLC, Non-small cell lung cancer

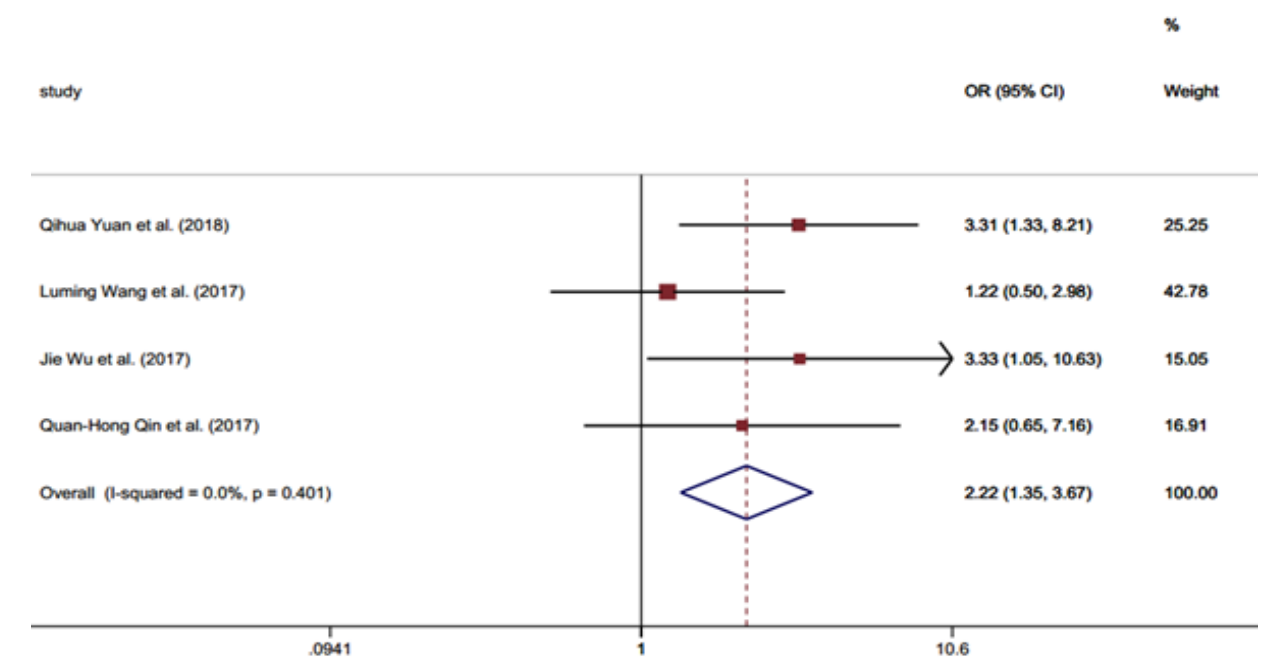

Figure 3. Forest Plot Illustrating the Association between the LINC01296 Expression and Tumor Stage

prognostic factors univariate analysis for OS in this study, showed a significant association between OS of patients with cancer and the LINC01296 expression and lymph node metastasis. Since, patients with high expression of LINC01296 compared with those had a low level of the expression, had a poor prognosis, the expression of LINC01296 might be considered as a potential biomarker in patients with cancer.
In a study, Anna Katharina Seitz et al.,(Seitz et al., 2017) identified aberrant expression of lncRNAs in 8 normal and 72 breast cancer samples using RNAsequencing. Among identified 89 lncRNAs that were significantly dysregulated, in vitro LINC01296 silencing reduced cell viability and migration. Moreover, they demonstrated that both LINC00958 and LINC01296 localized to both the nucleus and the cytoplasm and

Table 2. The Association between LINC01296 and Clinicopathological Criteria AND Univariate Analysis of Prognostic Factors for Overall Survival

\begin{tabular}{lccccc}
\hline & \multicolumn{3}{c}{ Correlation test } & \multicolumn{2}{c}{ Heterogeneity } \\
& OR & $95 \%$ CI & $P$-value & I2 (\%) & $P$-value \\
\hline Gender & 1.09 & $0.78-1.53$ & 0.592 & 0 & 0.956 \\
Age & 1.31 & $0.96-1.8$ & 0.086 & 0 & 0.556 \\
TNM stage & 2.67 & $1.83-3.88$ & 0 & 0 & 0.914 \\
Tumor stage & 2.22 & $1.34-3.66$ & 0.002 & 0 & 0.401 \\
Lymph node metastasis & 3.07 & $2.23-4.21$ & 0 & 45.6 & 0.065 \\
\hline & Univariate analysis & & Heterogeneity & \\
& HR & $95 \%$ CI & $P$-value & I2 (\%) & 0 \\
\hline Gender & 0.83 & -2.93 & 0.265 & 0.998 \\
Tumor stage & 2.66 & -5.76 & 0.071 & 65.7 & 0.054 \\
Lymph node metastasis & 2.39 & $1.16-3.63$ & 0 & 0 & 0.801 \\
LINC01296 expression (High vs Low) & 3.95 & $2.65-5.25$ & 0 & 26.1 & 0.248 \\
\hline
\end{tabular}




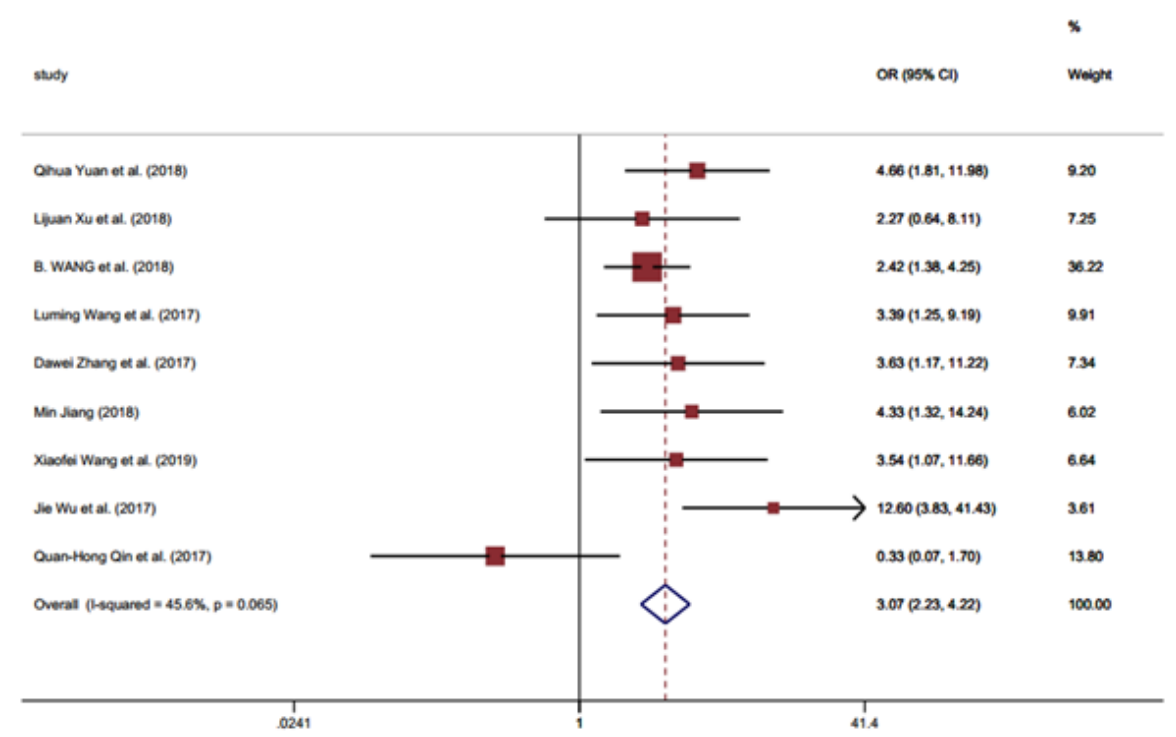

Figure 4. Forest Plot Illustrating the Association between the LINC01296 Expression and Lymph Node Metastasis

knock-down of them altered the expression of genes related to cell death/survival and cellular growth/ proliferation pathways. According to these findings, they suggested that LINC01296 act as oncogenes by stimulating proliferation and the metastatic process in breast cancer cells (Seitz et al., 2017). XIN YU et al., assessed the levels of LINC01296 expression in osteosarcoma cells in comparison to normal cells (Yu et al., 2018). Their findings revealed, the up regulation of LINC01296 was closely associated with the poor survival of osteosarcoma subjects. LINC01296 acts as an oncogenic factor which is able to increase the proliferation, invasion, and migration of osteosarcoma cells. Moreover, the expression of Cyclin D1 was positively correlated with the LINC01296 expression in osteosarcoma cells so that LINC01296 knockdown could lead to down regulation of cyclin D1 (Yu et al., 2018). It has been shown that Overexpression of LINC01296 is one of very important players in the progression of CRC(Qiu and Yan, 2015). In a Bing Liu et al., (Liu et al., 2018), shown that LINC01296 correlated with poor clinical prognosis in patients and the malignancy of CRC cell lines. Alteration of the LINC01296 expression impresses proliferation and metastasis of CRC cell and cause in vitro chemoresistance to 5-fluorouracil (5-FU). LINC01296 as a miR-26a direct target through LINC01296/miR-26a/GALNT3 axis encourage CRC malignancy using regulating O-glycosylated MUC1 by PI3K/AKT pathway. Upregulation of LINC01296 stimulates the in vivo tumorigenesis, liver metastasis and chemoresistance of CRC cell lines(Liu et al., 2018). Upregulation of LINC01296 was significantly seen in some another cancers such as gastric cancer, prostate cancer, urothelial carcinoma of the bladder and esophageal squamous cell carcinoma that in all of them correlated with lymph node metastasis, advanced TNM stage, and shorter overall survival(Wu et al., 2017; Qin et al., 2018; Wang et al., 2018; Wang et al., 2019).

LINC01296 knockdown using siRNAs could be related to inducing of cell apoptosis. LINC01296 could suppress cell apoptosis via targeting the Bcl-2/caspase-3 pathway(Yuan et al., 2019). Bcl-2 is known as a central regulator of cell life and death and caspase- 3 acts as an effector caspase which has a crucial role in cell apoptosis process (Evan and Littlewood, 1998; Mantena et al., 2006). In an epithelial-mesenchymal transition (EMT) process, a polarized epithelial cell by multiple biochemical changes accepts a mesenchymal cell phenotype. Gained features of this phenotype comprise enhanced capability of migratory, invasiveness, higher apoptosis resistance, and significantly increased ECM components production(Kalluri and Neilson, 2003). The loss of epithelial proteins such as E-cadherin and higher expression of nonepithelial markers such as N-cadherin and Vimentin are of the important features of EMT(Dong et al., 2012). Knockdown of INC01296 by reversing Snailmediated EMT decrease the capacities of invasiveness and migration. Taken together, these findings, the axis of LINC01296/Snail/ E-cadherin may have an important role in facilitating metastatic properties of pancreatic ductal adenocarcinoma(Yuan et al., 2019).

At the end of, some limitations of this meta-analysis comprise of following: 1) only 5 studies provide the survival data and only 1 study could provide the diseasefree survival data. 2) The sample size collected for overall survival analysis in this study was small that this subject does not provide statistical power to make a significant assessment.3) Also, implementation of subgroup analysis in one specific cancer or based on the ethnicity due to inadequate data was not possible. 4) Because of not doing an in vitro experiment, therefore, more studies for results confirmation of this meta-analysis are needed. In conclusion, this meta-analysis suggests that LINC01296 may be a diagnostic biomarker for shorter OS and metastasis.

In conclusion, cancer is one of very important public health problems which is associated with high rate mortality and mobility in patients with this disease. Finding, designing and developing new diagnostic platforms could contribute to increase survival rate of 
cancer patients. In this regards, a variety of biomarkers have been introduced by pre-clinical studies. Taken together, the results of this meta-analysis suggest that the expression of LINC01296 might be considered as a potential biomarker in patients with cancer.

\section{Acknowledgments}

Not applicable.

Ethics approval and consent to participate Not applicable.

\section{Contribution}

Conceptualization: FS, PKH, and YM.

Data curation: KT, MDF, PKH.

Formal analysis: KT, YM.

Funding acquisition: PKH.

Methodology: YM, KT.

Project administration: PKH, FS.

Visualization: ES, YM, and PKH.

Writing - original draft: MDF, ES, FS, and KT.

Writing - review \& editing: YM and PKH.

\section{Abbreviations}

ncRNAs: non-coding RNAs

microRNAs: Short ncRNAs

lncRNA: long ncRNAs

PCGEM1: prostate cancer gene expression marker 1

SPRY4-IT1: SPRY4 Intronic Transcript 1

PANDA: P21-associated ncRNA DNA damage -

activated

RoR: lincRNA-regulation of reprogramming

LINC01296: Long intergenic non-protein-coding

RNA 1296

TNM: Tumour, Node, Metastasis

PRISMA: The Preferred Reporting Items for

Systematic Reviews and Meta-analysis

MeSH: Medical Subject Headings

OS: overall survival

Competing Interests

The authors declare no conflict of interest.

\section{References}

Bartonicek N, Maag JL, Dinger ME (2016). Long noncoding RNAs in cancer: mechanisms of action and technological advancements. Mol Cancer, 15, 43.

Derrien T, Johnson R, Bussotti G, et al (2012). The GENCODE v7 catalog of human long noncoding RNAs: analysis of their gene structure, evolution, and expression. Genome Res, 22, 1775-89.

Dong C, Wu Y, Yao J, et al (2012). G9a interacts with Snail and is critical for Snail-mediated E-cadherin repression in human breast cancer. J Clin Invest, 122, 1469-86.

Evan G, Littlewood T (1998). A matter of life and cell death. Science, 281, 1317-22.

Jiang M, Xiao Y, Liu D, et al (2018). Overexpression of long noncoding RNA LINC01296 indicates an unfavorable prognosis and promotes tumorigenesis in breast cancer. Gene, 675, 217-24.

Jiang Y, Feng E, Sun L, et al (2017). An increased expression of long non-coding RNA PANDAR promotes cell proliferation and inhibits cell apoptosis in pancreatic ductal adenocarcinoma. Biomed Pharmacother, 95, 685-91.

Kalluri R, Neilson EG (2003). Epithelial-mesenchymal transition and its implications for fibrosis. J Clin Invest, 112, 1776-84.

Kung JT, Colognori D, Lee JT (2013). Long noncoding RNAs: past, present, and future. Genetics, 193, 651-69.

Kurozumi S, Yamaguchi Y, Kurosumi M, et al (2017). Recent trends in microRNA research into breast cancer with particular focus on the associations between microRNAs and intrinsic subtypes. J Hum Genet, 62, 15.

Liu B, Pan S, Xiao Y, et al (2018). LINC01296/miR-26a/ GALNT3 axis contributes to colorectal cancer progression by regulating O-glycosylated MUC1 via PI3K/AKT pathway. J Exp Clin Cancer Res, 37, 316.

Liu T, Chi H, Chen J, et al (2017). Curcumin suppresses proliferation and in vitro invasion of human prostate cancer stem cells by ceRNA effect of miR-145 and lncRNA-ROR. Gene, 631, 29-38.

Mantena SK, Sharma SD, Katiyar SK (2006). Berberine, a natural product, induces G1-phase cell cycle arrest and caspase-3-dependent apoptosis in human prostate carcinoma cells. Mol Cancer Ther, 5, 296-308.

Moher D, Liberati A, Tetzlaff J, et al (2009a). Preferred reporting items for systematic reviews and meta-analyses: the PRISMA statement. Ann Intern Med, 151, 264-9.

Moher D, Liberati A, Tetzlaff J, et al (2009b). Preferred reporting items for systematic reviews and meta-analyses: the PRISMA statement. PLoS Med, 6, e1000097.

Monteleone NJ, Lutz CS (2017). miR-708-5p: a microRNA with emerging roles in cancer. Oncotarget, 8, 71292.

Ponting CP, Belgard TG (2010). Transcribed dark matter: meaning or myth?. Hum Mol Genet, 19, 162-8.

Qin Q-H, Yin Z-Q, Li Y, et al (2018). Long intergenic noncoding RNA 01296 aggravates gastric cancer cells progress through miR-122/MMP-9. Biomed Pharmacother, 97, 450-7.

Qiu J-j, Yan J-b (2015). Long non-coding RNA LINC01296 is a potential prognostic biomarker in patients with colorectal cancer. Tumour Biol, 36, 7175-83.

Seitz AK, Christensen LL, Christensen E, et al (2017). Profiling of long non-coding RNAs identifies LINC00958 and LINC01296 as candidate oncogenes in bladder cancer. Sci Rep, 7, 395.

Siegel R, Ma J, Zou Z, et al (2014). Cancer statistics, 2014. CA: a cancer journal for clinicians, 64, 9-29.

Srikantan V, Zou Z, Petrovics G, et al (2000). PCGEM1, a prostate-specific gene, is overexpressed in prostate cancer. Proc Indian Natl Sci Acad, 97, 12216-21.

Wang L, Meng D, Wang Y, et al (2018). Long non-coding RNA LINC01296 promotes esophageal squamous cell carcinoma cell proliferation and invasion by epigenetic suppression of KLF2. Am J Cancer Res, 8, 2020.

Wang X, Wang L, Gong Y, et al (2019). long noncoding rna linc 01296 promotes cancer-cell proliferation and metastasis in urothelial carcinoma of the bladder. Onco Targets Ther, 12,75 .

Wells G (2001). The Newcastle-Ottawa Scale (NOS) for assessing the quality of non randomised studies in meta-analyses. http://www. ohri. ca/programs/clinical_ epidemiology/oxford. asp.

Wu J, Cheng G, Zhang C, et al (2017). Long noncoding RNA LINC01296 is associated with poor prognosis in prostate cancer and promotes cancer-cell proliferation and metastasis. Onco Targets Ther, 10, 1843.

Xu L, Wei B, Hui H, et al (2019). Positive feedback loop of 1ncRNA LINC01296/miR-598/Twist1 promotes non-small cell lung cancer tumorigenesis. $J$ Cell Physiol, 
234, 4563-71.

Xu Y, Leng K, Li Z, et al (2017). The prognostic potential and carcinogenesis of long non-coding RNA TUG1 in human cholangiocarcinoma. Oncotarget, 8, 65823.

Xu Y, Yao Y, Jiang X, et al (2018). SP1-induced upregulation of 1ncRNA SPRY4-IT1 exerts oncogenic properties by scaffolding EZH2/LSD1/DNMT1 and sponging miR-101$3 p$ in cholangiocarcinoma. J Exp Clin Cancer Res, 37, 81.

Yu X, Pang L, Yang T, et al (2018). IncRNA LINC01296 regulates the proliferation, metastasis and cell cycle of osteosarcoma through cyclin D1. Oncol Rep, 40, 2507-14.

Yuan Q, Zhang Y, Feng L, et al (2019). Upregulated long noncoding RNA LINC01296 indicates a dismal prognosis for pancreatic ductal adenocarcinoma and promotes cell metastatic properties by affecting EMT. J Cell Biochem, 120, 552-61.

This work is licensed under a Creative Commons AttributionNon Commercial 4.0 International License. 